\title{
Information as Order Hidden within Chance: An Application to Biology
}

\author{
Strumia A* \\ Istituto Nazionale di Alta Matematica "Francesco Severi", Italy \\ *Corresponding author: Alberto Strumia, Istituto Nazionale di Alta Matematica \\ "Francesco Severi", Italy, Email: alberto.strumia@icloud.com
}

\section{Review Article \\ Volume 3 Issue 3}

Received Date: August 12, 2019

Published Date: August 27, 2019

\section{Abstract}

We show, by didactical examples, how algorithmic information (coded e.g., into a computer program) is required to build the structure of an organized system (either simple or complex). Ordered structures can be obtained as attractors both by some dynamics starting from sequential initial conditions (order from order) and by some dynamics starting from random initial conditions (order from chance) provided that a leading algorithmic information is assigned to govern the evolution of the generating process. In absence of information emergence of some ordered structure, like e.g., an organ of a living system is so highly improbable to be impossible in practice. We provide didactical examples of static models of a human heart, each generated starting either from ordered initial conditions, or from random sparse initial conditions, or more realistically by random cellular automata (so that any mother cell is allowed to generate a daughter cell only in a random contiguous location). Significantly, as it was pointed out by Gregory Chaitin, not all algorithmic information can be compressed into a string shorter than the sequence of its original individual code digits (incompressible information string). A question is still open about the DNA and, more generally, any biological information: is it to be considered as a compressible or an incompressible code string? In our example of anatomic human heart model we have treated the sequence of the co-ordinates of each sphere (roughly modeling a cell) as an uncompressed string, while a compressed program string seems to be able to provide only less realistic models.

Keywords: Chance-order; Algorithmic information; Cellular automata; Computer models for biology

\section{Introduction}

"There is no science on singulars" (Latin, "scientia non est de singularibus" [1]), because human science is a knowledge through universals. In fact the human intellect acquires its knowledge abstracting from matter the universal form organizing each singular body made of "matter". So our mind, being "immaterial", does not know singulars, while our senses do, being "material" as part of our body made of "matter". Surprisingly the latter principle, which was well known by Aristotle and mediaeval authors like Thomas Aquinas and his followers, seems to be attained in some way, at least in some of its aspects and through a different way, by our contemporary logicians, mathematicians and experts of information theory. Knowing universally, in terms of our informational logic, appears to mean the capability to find a law, or an algorithm, the string code of which is shorter than the list of all individual entities when they are singularly collected in a set. So modern science seems to 


\section{Physical Science \& Biophysics Journal}

have rediscovered, in some sense, the ancient Aristotelian-Thomistic principle according to which not all the entities may be described (logic, cognition, science) or built (ontology, metaphysics, physics) by an algorithm (string shorter than the list of individuals). In fact there are entities, the string describing which cannot be other than the list of each single entity (incompressible string). Or, in terms of propositions, not any proposition (string) is decidable (by means of a theorem) within an axiomatic system (undecidability), since it cannot be reduced to the string of the axioms, according to the well-known Gödel's undecidability theorem [2]. Only a divine mind can know all singular details of an entity. While our human mind knows through universals, so it cannot find an algorithm describing all entities (whole theory or theory of everything) and all aspects of each of them. The sequence of such singular elements of a whole appears to us as completely random, since we cannot - because of principle reasons and not only because of technical difficulties - deduce by a rule (algorithm) any of the next element starting from the knowledge of the previous ones. But the datum of the incompressibility of a string, which we perceive as randomness, does not mean nonsense of that string, but simply that it is self-explained being reason to itself and therefore it needs no further explanation, being a fundamental law, even though rather complex. As Gregory Chaitin has observed: "for example, a regular string of $1 \mathrm{~s}$ and 0 s describing some data such as $0101010101 \ldots$ which continues for 1000 digits can be encapsulated in a shorter instruction "repeat 01500 times". A completely random string of digits cannot be reduced to a shorter program at all. It is said to be algorithmically incompressible" [3]. That notwithstanding, in some relevant and not so rare circumstance, the whole may reveal an order and an organized structure, capable to perform special activities (operations) as it happens, e.g., in biological living systems, or even in some physical and chemical complex systems. At present we do not know any compressed string (law or algorithm) capable to generate the actual sequence of the genetic code of a living being and we are compelled to list its individual elements one after the other as if they were provided randomly by nature. Something similar happens in the context of arithmetic when we deal with prime numbers, the sequence of which appears randomly distributed into the ordered set of natural numbers. An intensive discussion is animating the scientific world about the logical consistency of the idea itself of a theory of everything. A relevant example of different opinions about the matter is offered, e.g, by the contemporary debate between Stephen Wolfram [4] and Gregory Chaitin [5]. Wolfram is convinced that "in the end it will turn out that every detail of our universe does indeed follow rules that can be represented by a very simple program - and that everything we see will ultimately emerge just from running this program" [4]. Wolfram's conviction seems to arise by his deep experience with cellular automata, which may evolve into very complex structures, even being governed by very simple algorithmic rules. "In the existing sciences whenever a phenomenon is encountered that seems complex it is taken almost for granted that the phenomenon must be the result of some underlying mechanism that is itself complex. But my discovery that simple programs can produce great complexity makes it clear that this is not in fact correct. And indeed in the later parts of this book I will show that even remarkably simple programs seem to capture the essential mechanisms responsible for all sorts of important phenomena that in the past have always seemed far too complex to allow any simple explanation. It is not uncommon in the history of science that new ways of thinking are what finally allow longstanding issues to be addressed. But I have been amazed at just how many issues central to the foundations of the existing sciences I have been able to address by using the idea of thinking in terms of simple programs" [4]. While on the contrary Chaitin considers random strings (incompressible strings) as admissible in nature as indecidable propositions exist in an axiomatic system. "Wolfram has a very different view of complexity from mine. Wolfram's [4] view is that simple laws, simple combinatorial structures can produce very complicated unpredictable behavior. $\pi$ is a good example. If you didn't know where they come from its digits would look completely random. In fact, Wolfram says, maybe the universe contain non randomness, maybe everything is actually deterministic, maybe it's only pseudo randomness. And how could you tell the difference? The illusion of free will is because the future is too hard to predict but it's not really unpredictable" [5].

In the present paper we try to show how some complex - even if relatively simple or simplified ordered structures may arise i) either by already ordered initial conditions, ii) or by random initial conditions, provided that a suitable information (law/algorithm) is assigned, governing the evolution of the system. When such law (shorter string) is not known the full list (uncompressed string) of the elements involved in the structure of the system must be assigned in order to simulate the generation process of the system either assigning sequentially ordered initial conditions or random initial conditions. We emphasize that the probability to reach a final ordered structure, simply starting from randomly 


\section{Physical Science \& Biophysics Journal}

assigned points, if some information coded in a suitable string (it does not matter if compressible or incompressible) is not provided, is practically zero in presence of a great number of elements as in our universe. So we could say that order (information) may be hidden within chance but not completely suppressed. Ordered structures appear as attractors towards which the generation process tends even when the initial conditions are chosen randomly within a basin of attraction. An intriguing investigation might be based on the conjecture that universe could be modeled as a huge attractor involving minor nested attractors [6] (like e.g., the galaxies) nested in turn with inner attractors along a chain reaching living individuals and so on, down to cells, molecules, atoms and elementary particles. At present we do not know if living systems and some physical complex systems are fully determined by algorithms (compressed strings) or, at least, some of their properties are governed by full lists of instructions (incompressible strings). But we may guess that the light differentiations among the bodies of individuals of the same species can be generated, when genetic code is identical (as it happens for identical twins), thanks to the random bifurcations own to the non-linearity of the laws the governing generation of each individual body. In our paper we limit ourselves (for obvious reasons of space. More examples can be found in Strumia A [7]) to show and discuss two examples of heart models (ordered structures) one is able to generate by simple computing programs, either coded into compressed strings, or into uncompressed data files. At present we do not know if those data files are to be considered incompressible or if they might be compressed in future thanks to a non-trivial algorithm, i.e., a global mathematical law and not by local shortcuts similar to those employed to reduce graphic files dimension. The exposition will be developed according the following order. Section 2 will remind didactically some of the nowadays topics about the evolution of the notion of information which was born in communication engineering and now has become relevant in physics and mainly in biology. On an interdisciplinary viewpoint some meaningful "philosophical" re lections by some authors will be quoted, adding also our own re lections, in order to compare contemporary notion of information and Aristotelian form. In section 3 we propose a sort of rough model (based on a compressed string) of a human heart external shape generation, cell by cell. In section 4 a more realistic (anatomic) heart external shape model of cell by cell heart generation by cellular automata is finally presented, based on a public data list (non-compressed string) one can find on the web. Some conclusive remarks end the paper.

\section{Today's Information towards Aristotelian form? New Perspectives on Information in Mathematics, Physics and Biology}

In this first section we intend to introduce some elementary notions about the increasingly meaningful role of information in the context of the biological sciences, starting from the early decades of the 21st century [8-10]. A role which seems to involve both the question on the evolution of species and the matter of the emergence of life. In a wider sense information is playing a relevant role in order emergence (self-organization) of the structure and the dynamics of physical and biochemical complex systems. Nowadays we can see biologists, non-linear systems physicists, computer scientists and philosophers collaborate in a same research group in order to investigate new simulation models and theories about emergent life, organ formation in a body and mutations of species. Most of these topics involve philosophical problems related to the possible and unavoidable quest for an ontological interpretation of such theories beside to suggest heuristic paths orienting the research. People are now especially interested in proposing some definition of information which is more fundamental and relevant than the traditional one arisen in the field of noise free communication engineering. Significant steps have been carried out thanks to the analogy recognized between information and negative thermodynamic entropy, when non-equilibrium thermodynamics of open systems exchanging matter energy and information with the environment was developed by several authors. In the latter context the emergence of ordered structures within the physical open thermodynamic systems, governed by a sort of theleonomic dynamics, has oriented the researches to test how such thermodynamical systems could provide models for biological organisms and life emergence. Meanwhile the non-linear mechanics of dynamic systems discovered the existence of the attractors, i.e. solutions towards which all the trajectories, the initial conditions of which belong to a suitable basin of attraction, tend for time increasing values. Such attractors may be stable or unstable depending of the parameters characterizing each of them and may switch from stability to instability in correspondence to the parameters value switching. A comparison between a similar behavior and the change from life to death of a living system was considered as straightforward. Moreover some properties of a nonlinear system appeared as global (holistic) and not reducible to a sort of summation of more elementary local (reductionistic) properties. So the idea that some information characterizing the structure and the 


\section{Physical Science \& Biophysics Journal}

dynamics of the whole, which is not deducible starting from the properties of its single parts as if they were independent of the whole, suggested quite naturally to compare our contemporary notion of information with the ancient but always fascinating notion of Aristotelian form. Those ideas have been applied also to the species of living beings and not only the individual and the questions, i) if a sort of information may somehow orient the evolution of species, involving attractors and repellers, even if the initial conditions are determined by chance, ii) or if only chance and natural selection are enough to explain evolution. At present two schools of thinking are in competition [8].

The former school defends a neo-Darwinian position according to which the only random genetic mutations are enough to explain an evolution improving the qualities of species by spontaneous emergence of new information.

The latter, on the contrary, suggests that chance may not be enough to explain a gain (evolution) in the level in species, since an adequate cause is required in order to activate the emergence of new information from the potentiality of matter [8] as, in a greatly different historical and cultural context Aristotele proposed.

Therefore an increasing interest in Aristotelian doctrine of form appears today no more so peregrine as it was only until some decades ago. Surprisingly experimental investigations and mainly computer simulations provide relevant results supporting the ideas of the second stream of thinking. In fact simulations show that the great majority of random mutations are not of advantage for the species since they do not improve the ability to survive of the mutant individuals and only very few do. Moreover a sort of increasing genetic entropy accompanies mutations which destroys information rather than increasing it. A situation resembling the behavior of thermodynamic entropy the increasing of which, according to the second principle, decreases the power of heat in order to be transformed into mechanical work. Random genetic mutations cause more disorder (loss of information) than order (organization). Moreover the mutations result not to be genetically permanent, since they disappear in the descendants after few generations. In practice it has been shown that a threshold (minimum number of mutant individuals) exists under which the effect of mutations (either damaging or improving) extinguish after few generations [11].
Computer simulations, at least until now (for instance we may mention computer programs like Tierra, Mendel and Avida simulating random mutations involved in species evolution[12-13]) has provided results which seem not to be favorable to a merely random mechanism of a process improving the species. Then the researchers have been induced to examine in more depth the notion of information as a new immaterial factor playing an essential role, even if not yet well understood, either in governing the evolution of species and the birth of life and the emergence of an ordered structure in complex systems. In this framework at least two main problems arise.

How to define information and how to try to provide a model of information behavior?

Which is the cause of emergence of information in material systems (i.e., systems carrying mass and energy)? (according to an Aristotelian way of speaking we could say: which is the adequate cause of the eduction of the form from matter potency?).

The reductionistic and materialistic approach attempting to explain information as a mass-energy phenomenon, identifying it with its material carrier, has been just universally recognized as inadequate to describe experience. As a matter of fact we experience every day how information can be transferred from some material support to any other one, without alteration of its informational content. Since the early times of telecommunications and cybernetics it appeared as evidence what Norbert Wiener (1894-1964), one of the fathers of information theory, said: "Information is information, not matter or energy. No materialism which does not admit this can survive at the present day" [14].

\section{The Algorithmic Information and the Aristotelian Form}

We can easily recognize an increasing progression along the history of the attempts to achieve a proper definition of information. Starting from the early purely descriptive definitions, based on a physical and statistical approach as it was suggested by a comparison with thermodynamics and statistical mechanics, further steps were made in order to formulate more abstract and causally explicative definitions. In literature we may find references at least to the following kinds of theories of information and related definitions: i) the classical theory of information $[8,15]$, ii) the theory of specified complex information [8], iii) the algorithmic theory of information [8] iv) the universal theory of information [16]v) the pragmatic theory of information which is concerned to 


\section{Physical Science \& Biophysics Journal}

the cost of the machineries and networks required to process information [17]. Here we are interested especially in the algorithmic information about which we will emphasize some characters relevant also for biology. At present it seems to me that the approach of the algorithmic theory of information, adequately enriched by a semantic interpretation and content, is the most promising one, for the development of a mature scientific theory of information contributing to physics of complex systems and biology, and even to philosophy. The theory of algorithmic information, proposed and enriched by Ray Solomonoff (1926-2009), Andrej Nikolaevič Kolmogorov (1903-1987) and Gregory Chaitin (b. 1947), is concerned with complexity - as it is suitably defined within the theory itself - of the symbols involved in data and object structures. First of all a definition of algorithm is required. Adopting a very simple and didactical approach we can say that "An algorithm is a sequence of operations capable of bringing about the solution to a problem in a finite number of steps" [18]. Such definition is enough wide to host different kinds of algorithms involving different levels of information, progressively approaching to the Aristotelian notion of form. We will examine, by means of some examples, the methodological and epistemological relevance of the corresponding different levels and some implications for biology, foundation theory and even philosophy.

\section{Some Examples of Algorithms}

We limit ourselves to three simple well known examples of algorithm emphasizing the different level of information involved in each one.
The first level consists in a simple sequence of operations to be executed in order to solve some problem. In this case the kind of information involved is merely operational and does not imply any sort of definition of some entity. On an Aristotelian-Thomistic point of view it looks like the description of an accidental mutation of some entity built as a cluster (aggregate) of substances which is not endowed with a unique substantial form.

The second level, as we will see, is ontologically more relevant, since it actually defines an entity determining its structure. Philosophically we can say that the information involved in the algorithm properly defines the essence of an entity, just as an Aristotelian form.

The third level also defines an entity characterizing the dynamics which generates its structure, rather than defining immediately the structure as a whole. According to the Aristotelian-Thomistic terminology we say that the information involved in the algorithm specifies the nature of the generating information. Let us now briefly examine those examples.

\section{Algorithm to Exchange the Liquid Contained in two different Glasses: Let us consider two glasses, say A and $B$, filled respectively of water and wine. We want to transfer the water from $A$ into $B$ and vice versa.}

$$
A, B \rightarrow B, A
$$

The problem is easily solved with the aid of a third empty glass $\mathrm{C}$. Then the required algorithm is the following (Figure 1):

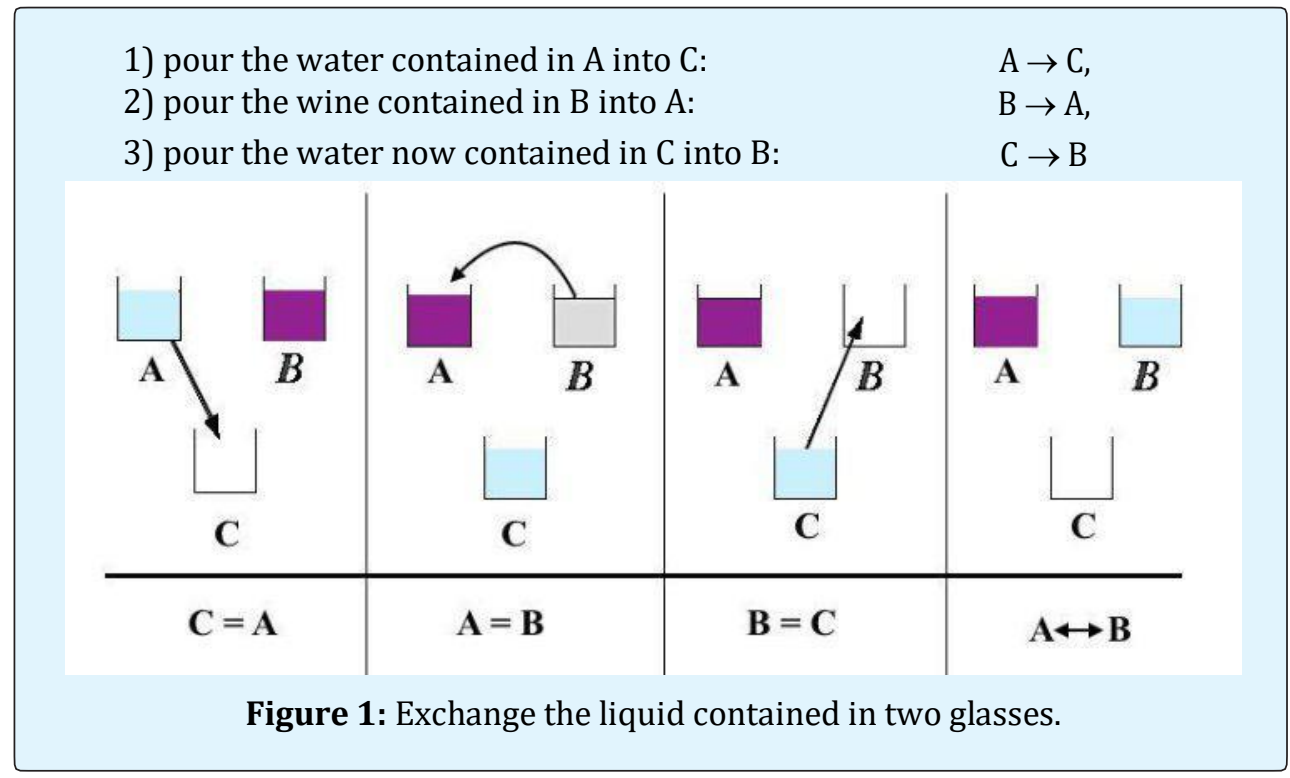

Strumia A. Information as Order Hidden within Chance: An Application to Biology. Phy Sci \& Biophy J 2019, 3(3): 000126. 


\section{Physical Science \& Biophysics Journal}

At the end of the procedure the desired exchange will result. The water which was into $A$ will have been transferred into $B$ and the wine originally in $B$ will be now in A. The algorithm, simply, describes an operative procedure which provides a mutation (becoming), while it does neither define nor give consistency (being) to an entity. Let us now examine a second kind of algorithm which, on the contrary, is actually able to define the structure (essence) of a new entity.

Algorithm to Generate a Fractal: Roughly speaking we can characterize a fractal as an infinitely rippled curve or surface the level of complexity of which is preserved at any magnification scale (fractals are more precisely classified considering their fractal dimension, a measure of the fraction of plane or space they ill when they are considered as wholes. One may see, e.g. [19] beside several papers and books one can find in literature with astonishing pictures of fractals as [20]). What is remarkable is the circumstance that the mathematical computation generating a fractal, besides providing an operational procedure, properly defines and in the same time actuates constructively its entity. Among all fractals we choose here, as an example, a typical Julia set (the dragon). The algorithm is the following:

We consider a complex number $\mathrm{z}_{0}=\mathrm{x}_{0}+\mathrm{i} \mathrm{y}_{0}$ the real part $\left(\mathrm{x}_{0}\right)$ and the imaginary part $\left(\mathrm{y}_{0}\right)$ of which run inside a suitable interval: [ 1 ; 1];
We choose another complex number $c=a+i b$ which is maintained constant along the whole procedure, as an identifier of the Julia set itself. In the example of Figure 2 we have set $c=0: 27334+i$ 0:00642;

We define a sequence of complex numbers $z_{n}=x_{n}+i y_{n} ; n$ $=0 ; 1 ; 2$; the initial term of which is just $\mathrm{z}_{0}$ and each next number is obtained adding $\mathrm{c}$ to the previous one squared. We have so the recurrence rule:

$$
z_{n+1}=z_{n}^{2}+c(1)
$$

We take the sum of a significantly high number of subsequent terms of the sequence (in principle the infinite series of all the terms of the sequence should be taken. In practice, on a computer a finite number of terms can be added. The greater is the number, the better will result the details in the picture).

At the end we evaluate the absolute value $h$ of the sum obtained:

$$
h=\left|\sum_{k=0}^{n} z_{k}\right|(2)
$$

If $h$ is greater than a suitable positive value $R$, before established, we paint on a computer display a pixel of coordinates $\left(\mathrm{x}_{0} ; \mathrm{y}_{0}\right)$ with a precise color (or gray level) of a suitable color map (or grayscale).

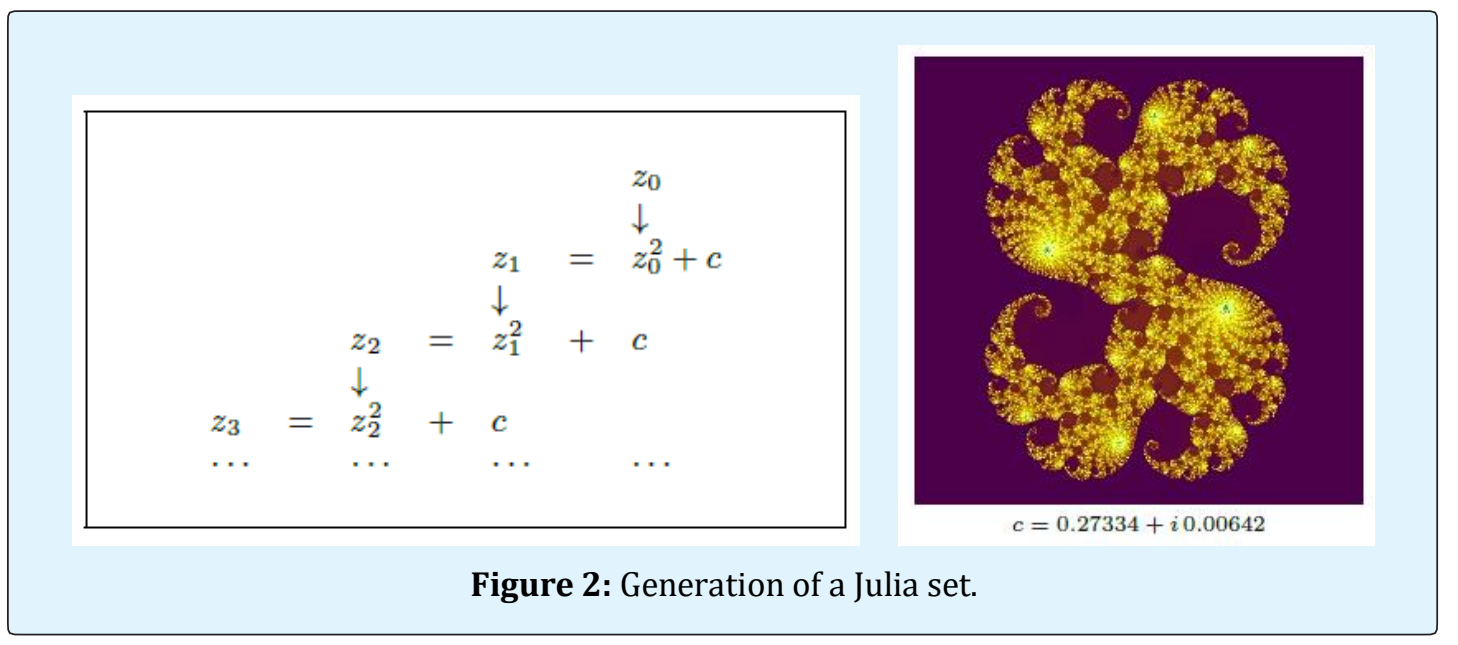

Manifestly the algorithm besides providing an operating procedure defines essentially the structure of a new entity, namely a Julia set, while constructing it.

\section{Algorithm to Determine a Fractal Basin of Attraction of a Chaotic Magnetic Pendulum: Our third example is}

provided by physics rather than mathematics. It consists also in a fractal set the structure of which results as an effect of the chaotic dynamics governing a magnetic pendulum driven by three magnets located in the vertices of an equilateral triangle. 


\section{Physical Science \& Biophysics Journal}

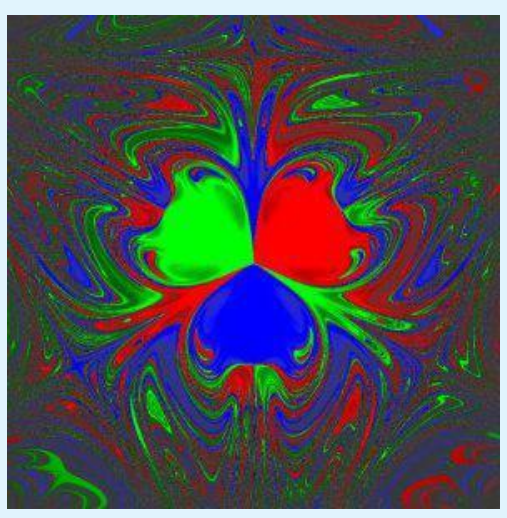

Figure 3: Fractal basin of attraction of a chaotic magnetic pendulum.

The motion of the pendulum appears to be random at all when it is observed at some time interval and with no regularity or order. Each trajectory seems to end onto one of the magnets without any choice criterion. That notwithstanding the dynamics is driven by a precise information arising from the laws of physics, since the arrival magnet depends exactly on the starting point from which the pendulum is initially released.

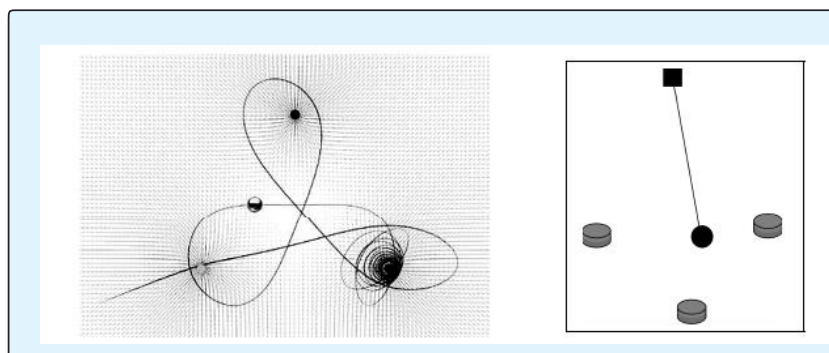

Figure 4: a) A chaotic trajectory of a magnetic pendulum; b) Sketch of a magnetic pendulum.

The pendulum dynamics being complex - determined by non-linear laws - it results to be strongly sensitive to the initial conditions. The starting point being even slightly displaced, the arrival magnet may change. So the basin of attraction (set of the initial conditions) related to the dynamics of the pendulum, exhibits a quite precise fractal structure. We point out that, in the present example the information which determines the fractal structure of the basin of attraction is determined through the dynamics of motion. Graphically the fractal basin is painted assigning distinct colors (or gray levels) dependent on the arrival magnet of the pendulum.

\section{Remark}

We want to emphasize, now, that people investigating algorithmic information are generally interested in defining the quantity of information involved into a computer program algorithm, which is viewed simply as a code string. Therefore a string program which solves some problem is considered as more rich of information as shorter is its code string. A matter involving a pragmatic instance of efficiency, minimizing time machine and then costs of program running. But it is known that not any problem is computable, since a string including an infinite number of characters, in many cases, cannot be compressed into a shorter one. Moreover also strings including a finite number of characters often cannot be compressed into a shorter one. In the language of set theory a similar circumstance arises because only a class of sets may be defined by a law (shorter string) according to which their elements are generated, thanks to the replacement axiom. All the remaining sets can be defined only listing their elements one by one (incompressible string). Within the frame of Gödel's theorem we can see the same problem as a matter of decidable propositions which correspond to a computable Gödel's number and undecidable propositions which are related to noncomputable Gödel's numbers. This is what one means when says that not all numbers are computable, since there does not exist a formula (shorter string) enabling us to evaluate all their digits avoiding to list them one by one. As a consequence, attaining physical dynamical systems, and especially biological and cognitive ones, we know that not all their activities are computable. So the irreducible qualitative and properly ontological aspects of their behavior has acquired a great relevance even on a scientific point of view beside their philosophical importance. Many of those non computable aspects concern information and related algorithms. A semantic approach seems now to be required beside the purely syntactic one developed in the classical information theory. Because the algorithm, as here is intended, is no longer simply identified by the string on which it is codedsum (whole) of the characters (parts) of which it is composed - rather being a definition, actualizing the dynamics of some resulting new entity. Rather such a definition is a logical law defining an entity and a sort of ontological form/information actuating its structure (essence) and its dynamics (nature). We emphasize that such a notion of algorithm, together with the previous philosophical interpretation seems to reveal a first but non-trivial rigorously scientific attempt to approach the definition/essence of the entity the structure/organization and the dynamics/nature of which are generated by the algorithm itself. We remember, in 


\section{Physical Science \& Biophysics Journal}

fact, that according to the Aristotelian-Thomisitic ontology the nature is just the essence as principle of acting ("Acting depends on nature, which is the principle of acting", Thomas Aquinas, In I Sent., Lib. 3, d. 18, q. 1, a. $1 \mathrm{co}$; "the word nature, so considered, appears to mean the essence of something, in order to its proper action", De Ente et Essentia, chapter 1, see[1]). So an algorithmic information involves more of philosophical content than some mere quantitative measure of information. Scientific investigation on information has become aware of this semantic exceeding contribution and is just attempting to grasp it with more and more suitable definitions. Information is recognized to be more than the length compression of a string of code. Among the first mathematicians who approached in a rigorous way the problem of characterizing the information, according to a careful comparison with the Aristotelian form, we have to mention René Thom (1923-2002), of whom we must cite at least his famous book [21]. In the frame of the mathematical physics of non-linear dynamical systems, for instance, a relevant approach to form/information has been developed following a methodology which is known as qualitative analysis of motion. Similar models are applied even in a biological context, in order to model the evolution of species or the emergence of self-organization during the transition from non-living matter to living organisms. All these research exhibit some non-trivial philosophical relevance since they investigate, as a matter of fact, the essence/nature of some entities by means of constructive definitions. Most likely more refined mathematical instruments will be required in order to formalize adequately information as a sort of algorithm and mathematics itself will widen as a true theory of entities (formal ontology). So information could involve both computable aspects and non-computable ones.

\section{Emergence and Evolution of Biological Information}

The relevant interest in the role of information in biology raises at least three main questions in the context of scientific research.

1. The first question is related to the emergence, or the origin of biological information. According to an Aristotelian terminology we should talk of eduction of the form from the potency of matter. So the problem for the search of an adequate efficient cause in order to obtain such an eduction arises each time a substantial mutation transforming some entity into another one happens in a stable way. In the contemporary scientific context this matter is often viewed as the problem of information production or information increment within some system (physical, biological, etc.). There is a tendency to guess that information may be produced or increased (at least locally) spontaneously, without an adequate causation, thanks to self-organization capability of the system itself, arising by random events.

2. A second question, which is strictly tied to the previous one, is related to the evolution of information, i.e., its mutation in time. In particular its spontaneous increment within some system, especially a living system.

3. The last question attains the problem of coding and copying biological information. Clearly biological information is no longer considered as residing only in the DNA code. Rather it appears as layered at several levels, even on the same biochemical, electro-chemical or, generally, physical medium.

The assumption that life complexity is only a spontaneous result of non-linearity of chaotic systems, has been shown to be incompatible with the numerical mathematical simulation models implemented on a computer, starting from their governing equations ([22] and related bibliography). "The explosion in the amount of biological information requires explanation" [23]. The useful non-ambiguous beneficial mutations (i.e., nondamaging at any level) arising from natural selection, result to be extremely rare. Chance seems not to be enough to generate improvements without an adequate cause [24]. On the contrary a process of loss of information (genetic entropy) is revealed, because of deleterious mutations which result to be the most likely mutations. So a sort of defensive barrier, conservative of complexity stability appears [13]. As to biological information coding scientists has observed that the genetic units consist in very precise instructions, coded in such a rich language that "any gene exhibits a level of complexity resembling that of a book" [23]. More languages (genetic codes) are present in the same genoma, with multiple levels (even three-dimensional), coding biological information, forming a network with several layers. Computer simulation models did not succeed in attempting to explain neither the emergence nor the increment of information, not withstanding both computer programs and the human genoma exhibit very resembling repetitive code schemes [25]. Information is responsible of organization and order emergence within the structure of a system, so that information increasing implies order increasing. What numeric simulation based on statistical mechanics and non-equilibrium thermodynamics-show, on the contrary, is that order is not spontaneously generated within the system, even if this latter is open (being able of exchanging matter and 


\section{Physical Science \& Biophysics Journal}

energy with the external environment). Information appears in a system, only in presence of a causal agent external to the system acting on it. "If an increase in order is extremely improbable when a system is closed, it is still extremely improbable when the system is open, unless something is entering which makes it not extremely improbable" [26]. The process of self-organization is activated thanks to the action of such an efficient/formal cause, which resembles to what, according to the Aristotelian-Thomistic theory is called eduction of a substantial form from the potency of matter. Starting from our recent knowledge on the physics of non-linear systems and the thermodynamics of non-equilibrium governing open dissipative systems, attempts are made in order to model the process of information emergence form matter (emergence of an organized structure in matter) by means of stable attractors. The dynamics of those attractors, not withstanding it appears chaotic and dominated by chance, is able to construct ordered structures. In fact the phase trajectories, solutions to dynamics, even starting at random from different initial conditions belonging to a basin of attraction (which may be even fractal), tend to ill precise regions of the phase space. So a whole arises from a confluence of parts, which are only apparently separated, being on the contrary non separable from the whole they are building, thanks to an information governing the structure and the dynamics of the process. Kaufmann's intuition that a new kind of notion of information, which is not merely statistical and syntactical, but involves also the semantic aspects seems to drive research towards the right direction. In particular the idea that some asymptotically stable attractor may be a good information carrier:

1. On one side ensures the presence of some information leading to structured order emerging within a system;

2. On the other side allows that chance play a wide role in the dynamics of the system, since the choice of initial conditions of the evolutive trajectories, within the basin of attraction, is left to chance without preventing that they all reach asymptotically the attractor itself.

So there does not exist any law in the arbitrary choice of the initial condition of the trajectories with the basin of attraction - the behavior of which may result even unpredictable if the attractor is chaotic - but some law exists within the dynamics of the system, involving some finality in its attractor solution. Such a finality (intended purpose) is typically a character of information. In principle several analogous levels of organization and finality may be obtained nesting several attractors into a hierarchy, so that some level of attractors is attracted in turn by a level of higher degree, until some first universal attractor is reached, which by definition cannot be attracted further, in order to prevent the occurrence of a logical paradox like that of the universal set. i) A lower level of organization could be, e.g., provided by a set of stable attractors representing the molecules, the dynamics of which is governed by ii) an immediately higher level of attractors organizing e.g., cells, the dynamics of which is ruled iii) by an higher level of attractors representing the organs of a living system; iv) a fourth level of attractors shapes the structure and the functionalities of individual living beings of different species; v) a fifth level of attractors will organize the species of living beings, and so on.

In principle one could guess, according to such a model of nested attractors [6], the existence of a chain starting at the level of the elementary particles and reaching the level of the universe as a whole. The chain is broken when some attractor lips from stability to instability, because of the occurrence of some accidental cause modifying the values of the parameters involved in the law of its level of dynamics. Then it happens that the second principle of thermodynamics, locally overcomes with the result of increasing disorder: the ordered organization of the system is partially damaged or fully destroyed. The whole scheme of chained attractors reminds a sort of fractal structure, even if it is not necessarily self similar in all its properties. At present research is open on these topics and a widened mathematics appears to be required resembling, at some levels, a sort of new version of ontology, suitably formalized.

\section{A Rough Heart Structure Model. Sequential, Random and Cellular Automata Generating Dynamics}

The aim of the paper is that of showing how ordered material systems (i.e., bodies of any kind) can be modeled as attractors towards which simpler elementary structures (particles, cells, etc.), even starting from random initial conditions, are driven by a suitable information (law, or sequence of laws). So order appears to arise from chance, while some suitable information is hidden within the random process itself. Several structures can be obtained assigning a relatively compact mathematical law, while more realistic models seem to require an uncompressed string listing all the components of the structure, as if the sequence of the respective numbers (co-ordinates of representative 


\section{Physical Science \& Biophysics Journal}

points of each elementary component) were noncomputable.

Programming codes are proposed in the Appendices.

\section{POV-Ray 3.7 Rendering of A Heart-Like External Structure}

We start with the simpler problem of approaching a 3D heart-like external shell by modifying the parametric equations of an ellipsoid, introducing a negative exponential deformation factor for $\mathrm{y}$ and an additional contribution $a y^{2}$ to $\mathrm{x}$, which seem to be enough for our purposes:

$$
x= \pm R \sin \theta \sin \phi+a y^{2}, y= \pm b R \cos \theta \sin \phi, z=c R \cos \phi e^{d \varphi}, \theta, \phi \in[0, \pi]
$$

a; b; c; d being suitable parameters. Rendering quality results to be significantly high if we implement the algorithm employing a ray tracing software like e.g., POVRay 3.7. We now examine the results when the heart model i) either is built as a whole, ii) or is generated by means of a random process, iii) or by a cellular automaton.

The Heart as a Whole: As a first step of our purpose we provide an algorithm to plot a rough sketch of a heart-like shape as a whole. Such an algorithm exhibits a final result but prevents to show the dynamics according to which a heart is generated step by step by cell multiplication (Figure 5).

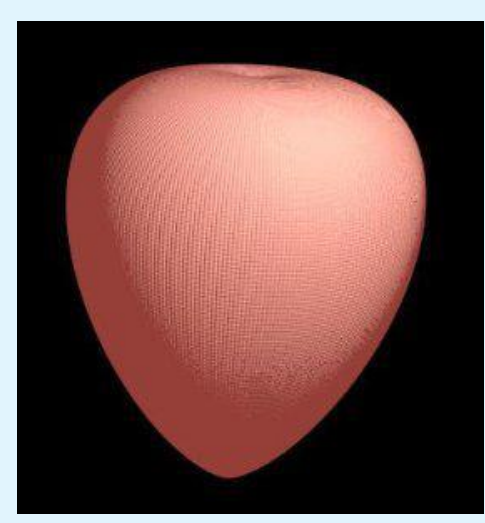

Figure 5: A rough sketch of a heart as a whole (POVRay 3.7 rendering).

(See POV-Ray 3.7 code to generate Figure 5 in Appendix A).

Sequential Dynamics Generating a Simple Heart-Like Structure (Order form Order): As a second step we show in the following pictures (Figures $6 \& 7$ ) the sequential sections of a progressive (point by point) longitudinal and latitudinal generation of the heart shape structure. We note that the graphic resolution, even if very refined, cannot reproduce the real scale of cell dimensions. Moreover such a highly ordered sequential dynamics is still unable to simulate a realistic cell multiplication process.

(See POV-Ray 3.7 code to generate Figures 6 and 7 in Appendix B).

Random Sparse Dynamics Generating a Simple HeartLike Structure (Order from Chance): A further passage consists in examining the random rendering steps of the algorithmic heart model which are illustrated in the following Figure 8. What is remarkable is that notwithstanding that each point is chosen by chance, an algorithmic information drives the dynamics towards an orderly structured attractor.
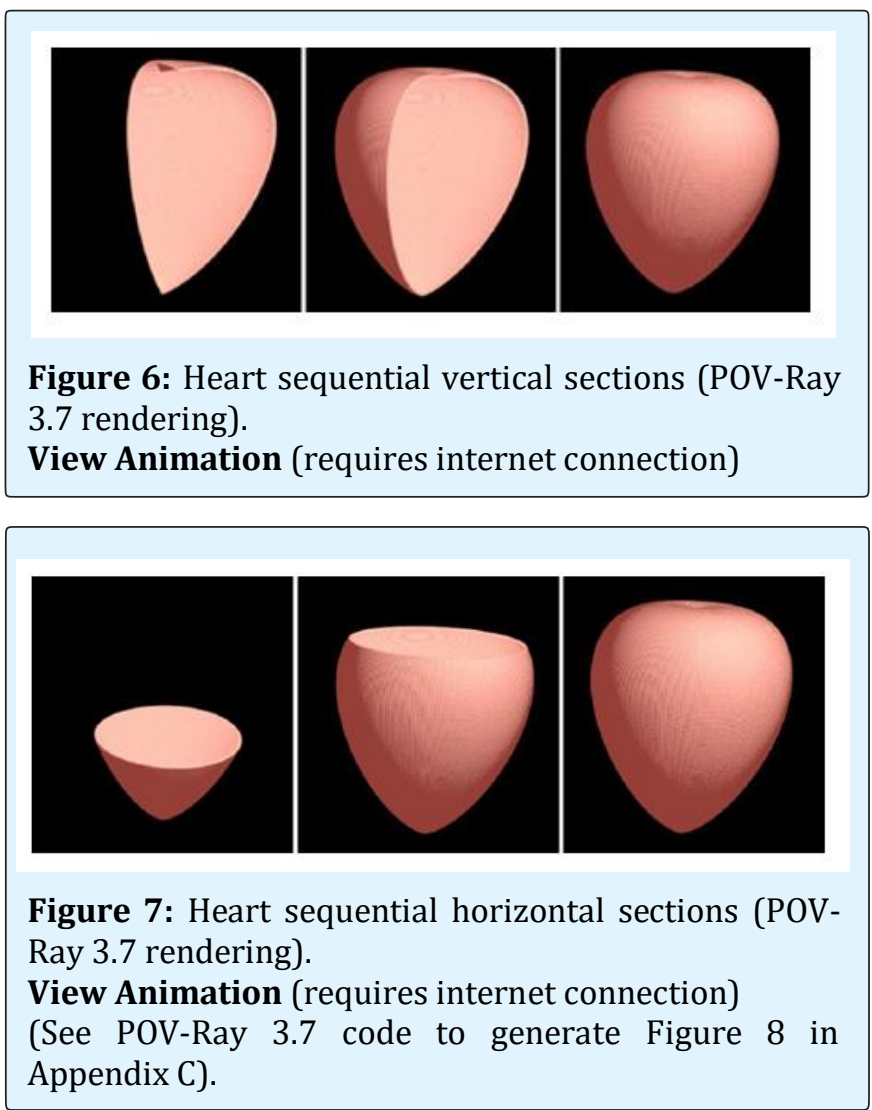

Random Cellular Automaton Generating A Simple Heart-Like Structure (Order From Chance): A sensibly more realistic dynamics can be obtained adding to the model proposed in the previous section the constraint that each mother cell may generate a daughter cell only in a contiguous position (as it is required for cellular 


\section{Physical Science \& Biophysics Journal}

automata). The result is shown in Figure 9 and related animation.

(See POV-Ray 3.7 code to generate Figure 9 in Appendix D).

\section{A Genuinely Realistic Model of Heart Structure. Sequential and Random Rendering Processes}

\section{Heart Model Based on POV-Ray 3.7 Smooth Triangle Object}

Bob Hughes has written, in year 2000, a 3D POV-Ray heart model code based on a list of data from a real human heart representation obtained by decomposition of the external heart surface into small smooth triangles (see Figure 10). The zipped code files can be downloaded at [27]. We point out that the latter author does not start from a mathematical law (compressed string) to calculate the data identifying the smooth triangles (which in POVRay are obtained interpolating a curved surface starting from the local gradient vectors assigned in each vertex of the triangle). So he needs to provide a full data list of the co-ordinates and local normal components of each individual smooth triangle. The data file (heart.inc) involves more than 1600 smooth triangles, the related data being listed sequentially one after the other as if the information were at all irreducible. An intriguing question is if similar biological data are truly non-computable (incompressible string of irreducible information) or we may guess that in future some shorter rule (law) could be discovered.

We emphasize that the code offers a nice representation of a heart external shape as a whole, but here we are interested in controlling each individual triangle and possibly each individual cell (modeled by a small sphere) in order to build either an ordered sequential or random or cellular automaton process, like those examined in the previous sections, in order to try to simulate somehow the cell multiplication dynamics.

(See POV-Ray 3.7 code to generate Figure 10 and some details on "smooth triangles" in Appendix E).

\section{Heart Structure Based on Single Cells in Ordinary Triangle Objects}

In order to be able to follow each cell, represented by a single point, belonging to some triangle we simplify the code by replacing smooth triangle objects by ordinary triangles. The heart external surface results no longer so perfectly smooth but we gain the advantage of a more realistic single cell multiplication representation. The resulting effect appears in each image representing a step of the generation process and even more when the images are collected into a movie (see Figure 11 and animation). (See POV-Ray 3.7 code to generate Figure 11 in Appendix F).

Generating a Heart Structure by an Ordered Sequence of Cells in Ordinary Triangles (Order Form Order): Ordinary triangles are characterized simply by the triplets of the co-ordinates of their vertices. So the "HeartPoints.inc" to be included by the POV-Ray 3.7 code can be obtained by the original "heart.inc" file

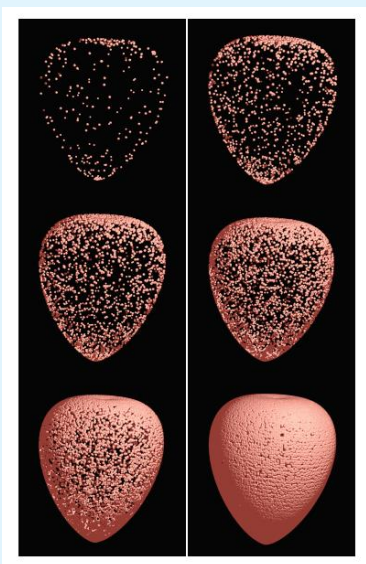

Figure 8: Heart random generation steps (POV-Ray 3.7 rendering).

View Animation (requires internet connection)

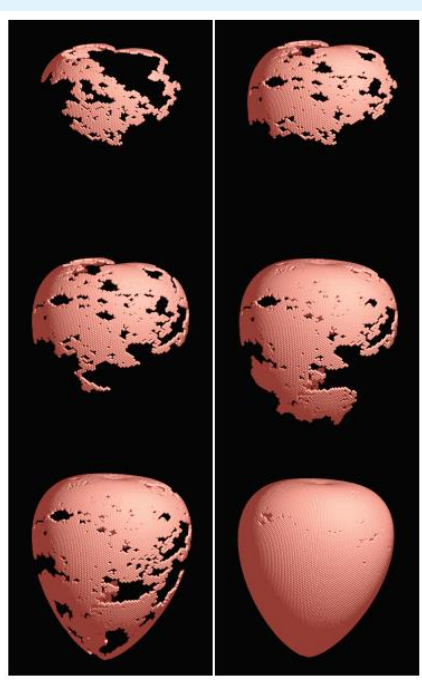

Figure 9: Heart generation steps by a cellular automaton (POV-Ray 3.7 rendering).

View Animation (requires internet connection) 


\section{Physical Science \& Biophysics Journal}

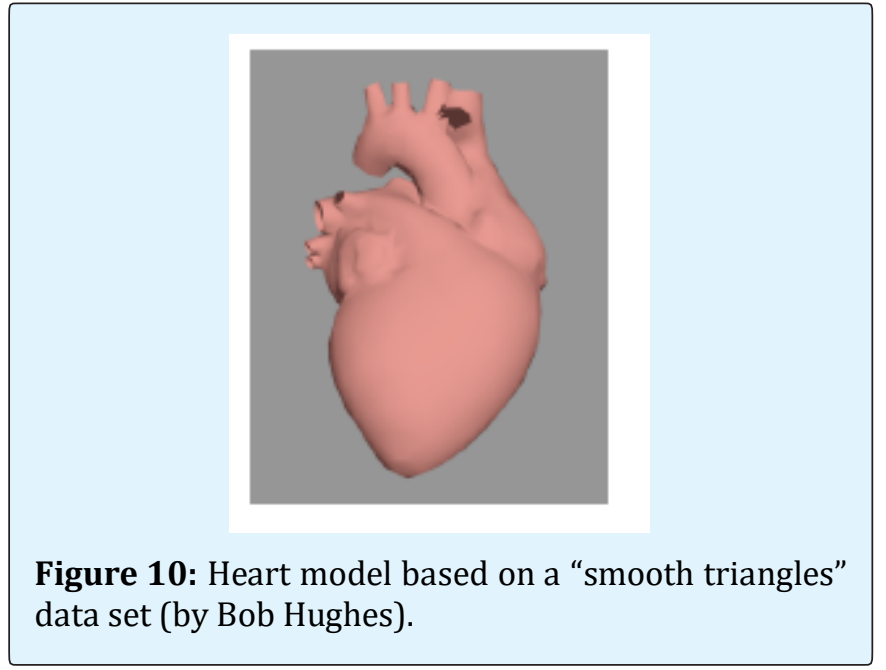

Defining the smooth triangles data simply dropping the second vector of each couple, which is related to the local normal vector.

(See POV-Ray 3.7 code to generate Figure 11 in Appendix F).

Generating a Heart Structure by Random Sparse Cells in Ordinary Triangles (Order form Chance): A random process generating the heart model point by point impressively shows how chance combined with information allows to build an ordered structure starting by initial conditions assigned randomly.

(See POV-Ray 3.7 code to generate Figure 12 in Appendix G).

\section{Generating a Heart Structure by Cells Generated by} Random Cellular Automata (Order Form Chance): As a final step we implement a random process generating the heart model by cellular automata. Of course the cell dimension cannot be realistic respect to true biological conditions. But the graphic result is enough to render the idea of what may happen in nature. Information seems just to drive chance towards order and organization.

(See POV-Ray 3.7 code to generate Figure 13 in Appendix $\mathrm{H})$.

\section{Conclusion}

In the present paper we have attempted to show how chance and information need to work together so that ordered structures, like complex systems and in particular living bodies, are allowed to emerge locally starting from unorganized matter. The role of some driving information has appeared essential in order to direct the evolutive trajectories of any system towards an organized ordered structure resulting as an attractor. Matter and energy alone prove to be not enough to generate order, because of the second law of

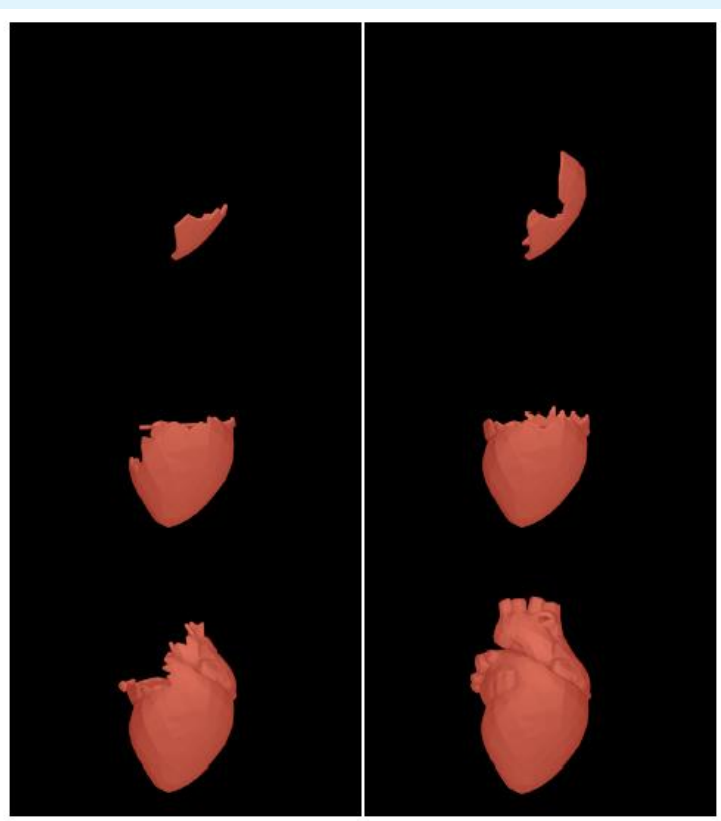

Figure 11: Heart generation steps by an ordered sequence of cells in ordinary triangles (POV-Ray 3.7 rendering).

View Animation (Requires Internet Connection)

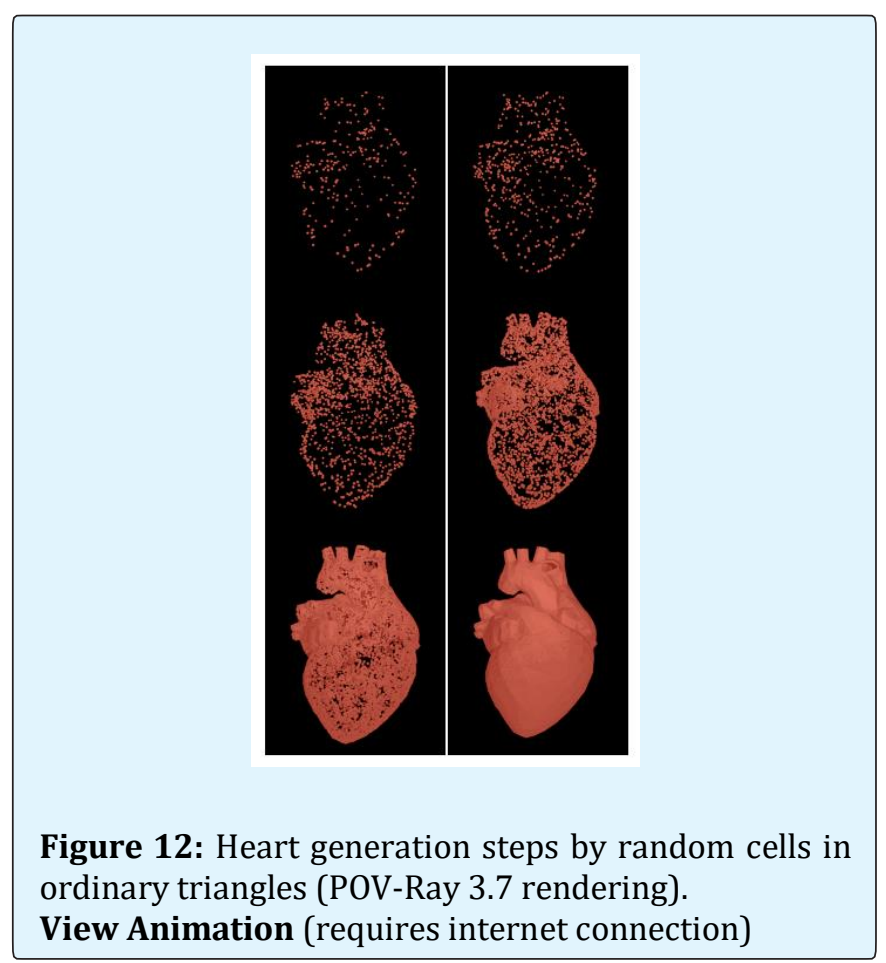




\section{Physical Science \& Biophysics Journal}

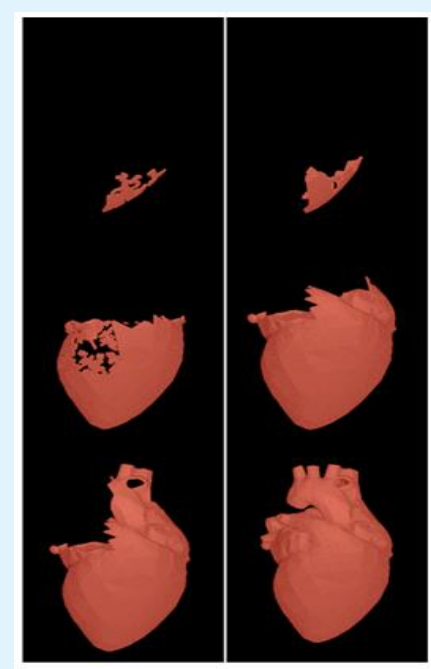

Figure 13: Heart generation steps by cells as cellular automata belonging to ordinary triangles (POV-Ray 3.7 rendering).

VIEW ANIMATION (requires internet connection)

Thermodynamics, which compels any matter-energy system towards disorder and thermal equilibrium. Even if, locally, some partially ordered structures may emerge, by chance, the probability of such an occurrence is very small and the entire age of the universe would not be enough to produce an organized system comparable to the living beings which actually we observe on our planet. The number of the ordered possible combinations of particles is too small compared with the huge number of disordered combinations. Moreover, even if an ordered configuration might occur by chance, its stability in time would be even more and more unlikely. A further governing principle like information, which is neither matter nor energy (according to $\mathrm{N}$. Wiener) seems to play an essential role in the process of order and organized systems emergence from matter. We have shown how algorithmic information (in the sense we have proposed just in sec. 2) can operate in order to generate complex systems either starting from an already ordered sequence of initial conditions or starting from random initial conditions, leading to the same geometry of the resulting objects, as attractors towards which the evolutive trajectories are led thanks to information. Of special interest, in relation to biological systems, has been revealed cellular automata since they add to the driving algorithmic information the constraint that any daughter cell is located in contiguity with its mother cell. No matter if the choice of the near location of the daughter cell is chosen by chance. What is relevant is the role of the law (information) according to which the daughter cell is born. The techniques implemented, have been applied to a biological system like a human organ, e.g., the heart, in order to simulate the generation or regeneration of its tissue by a stem cell. We saw how a simple program (compressed string) allows to obtain only a rough model of an heart shape, while a true realistic anatomic shape seems to require to know the full (uncompressed) list of the co-ordinates localizing the single cells, even if they are schematically represented by small spheres. An intriguing question arises if a biological organ belonging to a living body can be generated by an algorithm which can be compressed within a relatively short program string, or if an incompressible string of data is required to describe each single cell or constituent part of the whole system. Is the DNA, and more generally the biological code responsible of a living body generation, more resemblant to a compressible or to an incompressible string of code? In principle one could guess to model the whole universe as a set of nested attractors. In the present investigation we have limited ourselves only to attack the problem of the emergence of complex boundary geometrical shapes of bodies (like a living organ) thanks to the concurrence of some information (i.e., something resembling an Aristotelian form). A More intriguing matter would be, beside that of the generation of the external and internal organized structure of complex systems, that of modeling their behavior along time, i.e., their dynamics. So timidly approaching the matter of their nature (in the Aristotelian Thomistic sense of the word, i.e., operational ability), together with the matter of their essence (i.e., existing ability as organized ordered structures). Further researches will be required in future to widen the present program of investigating the role of information (form) as an immaterial principle of organization and activity of matter-energy. We hope that the INTERS project on "Form and information" may offer a suitable context to develop such a stimulating search and will be able to provide some more relevant results.

\section{References}

1. Thomas Aquinas, Index Thomisticus.

2. Godel K (2001) "On formally undecidable propositions of Principia Mathematica and related systems I". $1^{\text {st }}$ (Vol.), Collected Works, Oxford University Press, New York (NY), pp: 144-195.

3. Chaitin G (1992) Information theoretic incomplitness, World Scientific, Singapore. 


\section{Physical Science \& Biophysics Journal}

4. Wolfram S (2002) A new kind of science. Bulletin (New Series) of the American Mathematical Society 40(1): 143-150.

5. Chaitin GJ (1999) The unknowable. $1^{\text {st }}$ (Edn.), Springer-Verlag, Singapore, pp: 124.

6. Kaufmann SA (2014) Evolution Beyond Entailing Law: The Roles of Embodied Information and Self Organization. University of Vermont, pp: 513-532.

7. Strumia (2019) Chance and order: fractals, cellular automata and biology (year 2019).

8. Marks II RJ and Oth. editors (2014) Biological information. New perspectives, Proceedings of a Symposium, Cornell University, World Scientific, Singapore.

9. Strumia A (2017) Dalla filosofia della scienza alla filosofia nella scienza. Sisri-Edusc, Roma, pp: 360.

10. Strumia A (2019) Information, Recent Perspectives for the Natural Sciences.

11. Gibson PG, Baumgardner JR, Brewer WH, Sanford JC (2014) Can Purifying Natural Selection Preserve Biological Information? Southern Illinois University, pp: 232-263.

12. Ewert W, Dembski WA, Marks II RJ (2014) Tierra: The Character of Adaptation. pp: 105-138.

13. Nelson CW, Sanford JC (2014) Computational Evolution Experiments Reveal a Net Loss of Genetic Information Despite Selection. pp: 338-368.

14. Wiener N (1965) Cybernetics: or the control and communication in the animal and the machine, Technology Press, MIT, Cambridge (MA).
15. Shannon C (1948) A Mathematical Theory of Communication. The Bell System Technical Journal, 27: 379-423, 623-656.

16. Gitt W, Compton R, Fernandez J (2014) Biological Information. What is It? pp: 11-25.

17. Oller JW (2014) Pragmatic Information. pp: 64-86.

18. Sarti E (2002) Information.

19. Albert Strumia Galleria di frattali - Fractal gallery

20. Peitgen HO, Richter PH (2012) The Beauty of Fractals, Springer-Verlag, Berlin-Heidelberg-New York-Tokyo.

21. Thom R (1989) Structural stability and morphogenesis: an outline of a general theory of models, Perseus Books, Cambridge (MA).

22. Basener WF (2014) Limits of Chaos and Progress in Evolutionary Dynamics. pp: 87-104.

23. Sanford JC (2014) Introduction to the $2^{\text {nd }}$ meeting session. pp: 203-209.

24. Montañez G, Marks II RJ, Fernandez J, Sanford JC (2014) Multiple Overlapping Genetic Codes Profoundly Reduce the Probability of Beneficial Mutation. pp: 139-167.

25. Seaman J (2014) A Sequence Comparison between the Human Genome and Computer Code. pp: 384-401.

26. G. Sewell (2014) Entropy, Evolution and Open Systems. pp: 168-178.

27. The povray objects collections. 